Laboratory techniques

\title{
Use of monoclonal anti-actin as a megakaryocyte marker in paraffin wax embedded bone marrow biopsy specimens
}

\author{
C BOQUE, N PUJOL-MOIX, M A LINDE,* C MURCIA, C GUANYABENS, \\ J SOLER Servei d'Hematologia, ${ }^{*}$ Servei d'Anatomia Patològica, Hospital de la Santa Creu i Sant Pau, \\ Barcelona, Spain
}

SUMMARY Monoclonal anti-actin was used as a marker of megakaryocytes in Zenker's fixed, paraffin wax embedded bone marrow tissue, using an immunoperoxidase staining method. Twenty bone marrow samples were studied, including controls, and different myeloproliferative and myelodysplastic syndromes. The results were compared with those obtained using factor VIII related antigen (F VIII RAg) immunolabelling.

Anti-actin is as good a marker for megakaryocytes as anti-FVIIIRAg and is potentially clinically useful when morphological identification is difficult, when bone marrow aspiration is unsuccessful, or when quantitative evaluation of tissue sections is required.

Well differentiated and mature megakaryocytes are easily detected in bone marrow biopsy specimens but the identification of more primitive megakaryocytes may be impossible by conventional methods. Immunohistochemical labelling of bone marrow biopsy specimens is of particular interest in such cases.

Most of the antibodies that can detect megakaryocytes are not suitable for routine bone marrow biopsy specimens, ${ }^{12}$ because they recognise antigens destroyed during fixation, decalcification, and paraffin wax embedding. Antibodies to factor VIII related antigen (FVIIIRAg), however, have been used as markers of megakaryocytes in paraffin wax embedded bone marrow biopsy specimens and other tissue samples. ${ }^{34}$ In this paper we describe the use of an alternative monoclonal anti-actin with an immunoperoxidase method and compare the results between FVIIIRAg and actin immunolabelling in normal bone marrows and in several haematological abnormalities which affect megakaryocytes.

\section{Material and methods}

Of the 20 patients studied, four had normal bone marrow biopsy specimens (taken for the staging of solid tumours) and 16 had different haematological

Accepted for publication 16 February 1989 disorders affecting megakaryocytopoiesis: prima thrombocythemia $(\mathrm{PT}) \mathrm{n}=2$; policythemia rube vera $(P R V) n=2$; idiopathic myelofibrosis (MFF $n=4$; chronic granulocytic leukaemia $(C G L) n=4$; and myelodysplastic syndrome (MDS) $n=4$.

Bone marrow biopsy specimens were obtained by a $\stackrel{\mathbb{D}}{2}$ conventional needle biopsy technique, immediately $\overrightarrow{\vec{A}}$ fixed in Zenker's solution, and embedded in paraffin wax after decalcification in acetic acid.

Immunoperoxidase staining using a polyclonal antibody to human FVIIIRAg at a 1/800 dilution (Dakopatts, Denmark) and a monoclonal antibody to actin at a 1/2000 dilution (Amersham, UK) was done by the avidin-biotin method (Vectastain ABC Kit, Vector Laboratories, Burlingame, California, USA). Control samples were incubated with normal serum instead of primary antibody.

The results were evaluated by light microscopy, $D$ noting both the numbers of stained megakaryocytes and the intensity of their staining.

Results

Results of the immunoperoxidase staining for mono- $\bar{Q}$ clonal actin and for polyclonal FVIIIRAg are sum- $c$ marised in the table.

Selective staining of labelled megakaryocytes is $\stackrel{\oplus}{+}$ shown beside the haematotoxylin and eosin stain in 7 the figure. 

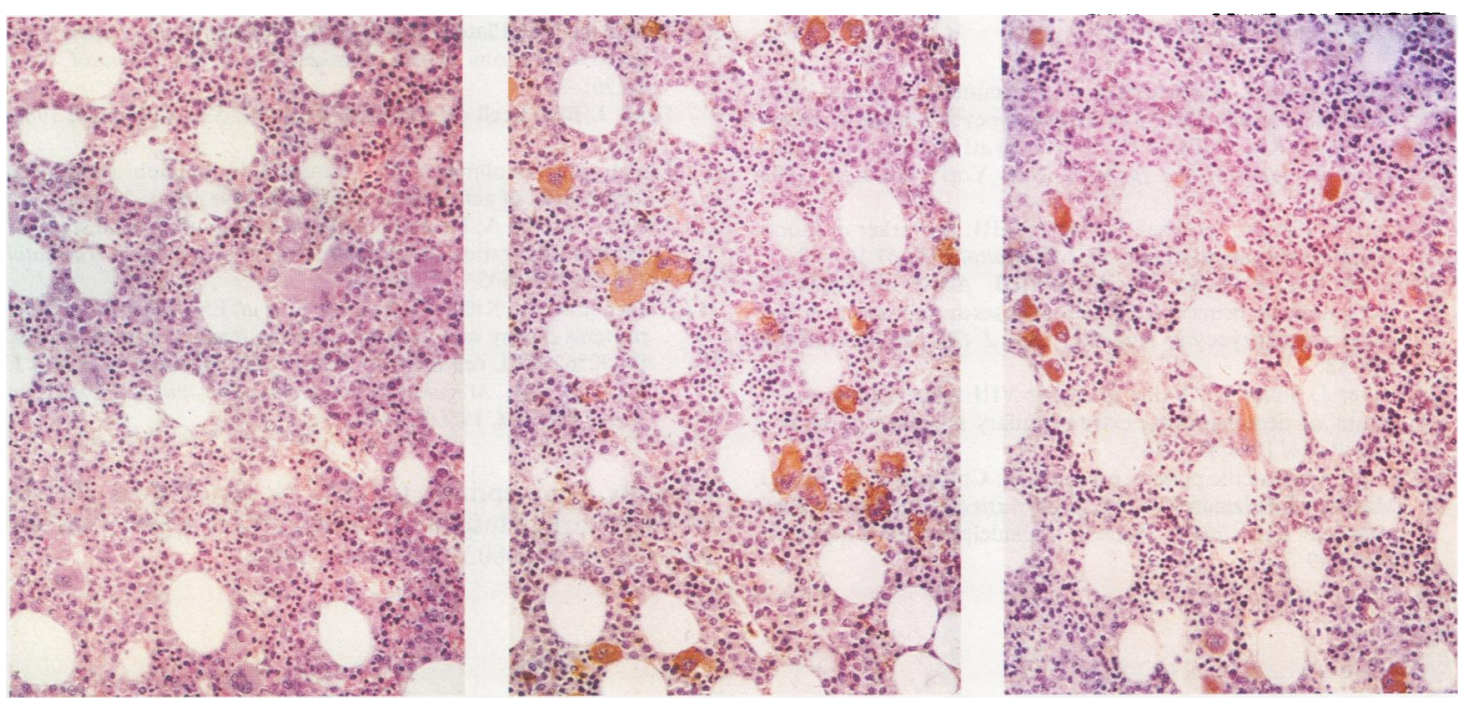

Figure Normal bone marrow. (a) Haematoxylin and eosin stain; (b) immunoperoxidase stain with monoclonal anti-actin;

(c) immunoperoxidase stain with polyclonal anti-FVIIIRAg.

\section{Discussion}

Actin is the principal protein of the platelet cytoskeleton and represents around $35 \%$ of the cell total protein content. ${ }^{5}$ It is synthesised by megakaryocytes and constitutes the contractile apparatus which regulates the shape changes occurring during activation of platelets. ${ }^{6}$

In our hands, actin proved a reliable marker for the megakaryocyte cell line in bone marrow samples, though this is, to the best of our knowledge, the first time the technique has been reported. Although it is known that many bone marrow cells contain actin ${ }^{7}$ only megakaryocytes are prominently stained by the method. Other structures that also stain for actin in bone marrow samples are muscle wall of the vessels, pericytes of the capillaries, and platelets. ${ }^{8}$
Megakaryocyte immunolabelling is clinically useful when difficulty arises in the morphological recognition of megakaryocytes because of immaturity and atypia, or when bone marrow aspiration is unsuccessful (dry tap), and when it is helpful to quantitate the megakaryocyte population accurately in tissue sections.

The intensity of the stain seems to depend on the degree of maturity and capacity of megakaryocytes for protein synthesis. Further studies should be done to evaluate the stage of megakaryocyte differentiation needed to stain with actin as has been previously done for FVIIIRAg. ${ }^{910}$

Actin is as good a marker for megakaryocytes as FVIIIRAg, and it can be helpful when a study of megakaryocytes is needed on paraffin wax embedded bone marrow samples.

Table Results of immunoperoxidase staining for monoclonal anti-actin and for polyclonal anti-FVIIIRAg antibodies

\begin{tabular}{|c|c|c|c|}
\hline Diagnosis & $\begin{array}{l}\text { No of } \\
\text { cases }\end{array}$ & Actin & FVIIIRAg \\
\hline $\begin{array}{l}\text { Control } \\
\text { PT } \\
\text { PRV } \\
\text { MF } \\
\text { CGL } \\
\text { MDS }\end{array}$ & $\begin{array}{l}4 \\
2 \\
2 \\
4 \\
4 \\
4\end{array}$ & $\begin{array}{l}++,++++,++++, \quad++++ \\
++++, \quad++++ \\
++++, \quad \\
+, \quad,++++, \quad++++ \\
+,+,++++,++++ \\
+,++++, \quad+++, \quad++\end{array}$ & $\begin{array}{l}++++,++++,++++,++++ \\
-,++ \\
+++,+ \\
++++,-, \quad,++++ \\
++++,+++,++++,++ \\
+,++++,++++, \quad+\end{array}$ \\
\hline
\end{tabular}

$++++=$ strong or $+++=$ moderate positivity in more than $75 \%$ of megakaryocytes.

$++=$ strong or + moderate positivity in between $25 \%$ to $75 \%$ of megakaryocytes.

- = less than $25 \%$ of megakaryocytes stained or a very weak positivity considered to be negative. 


\section{References}

1 Breton-Gorius $\mathrm{J}$, Vainchenker $\mathrm{W}$. Immunological and cytochemical characterization of megakaryocytic lineage leukemia. In: Levine RF, Williams N, Levin J, Evatt BL, eds. Megakaryocyte development and function. New York: AR Liss, 1986: $301-17$.

2 Adany R, Kiss A, Muszbek L. Factor XIII: A marker of monoand megakaryocytopoiesis. $\mathrm{Br}$ J Haematol 1987;67:167-72.

3 Hruban RH, Kuhajda FP, Mann RB. Acute myelofibrosis. Immunohistochemical study of four cases and comparison with acute megakaryocytic leukemia. Am J Clin Pathol 1987;88: 578-88.

4 Crocker J, Smith PJ. Value of factor VIII related antigen as a means of demonstrating extramedullary megakaryopoiesis. $J$ Clin Pathol 1984;37:834-5.

5 Cohen I. Contractile platelet proteins. In: Coldman RW, Hirst J, Marder VJ, Salzman EW, eds. Hemostasis and thrombosis. Basic principles and clinical practice. Philadelphia: JB Lippincott, 1982:459-71.
6 Nachmias VT. Platelet and megakaryocyte shape change: triggered alterations in the cytoskeleton. Semin Hematol 1983, 20:261-81.

7 Palek J. Blood cell cytoskeleton. I and II. Semin Hematol 1983; 20:1.

8 Mukai K, Schollmeyer JV, Rosai J. Immunohistochemical localization of actin. Am J Surg Pathol 1981;5:91-7.

9 Vinci G, Tabilio A, Deschamps JF, et al. Immunological study of in vitro maturation of human megakaryocytes. $\mathrm{Br} \mathrm{J} \mathrm{Haematol}$ 1984;56:589-605.

10 Vainchenker W, Kieffer N, Cramer E, et al. Expression of platelet proteins during maturation of human megakaryocytes and of the K562/HEL cell lines. In: Levine RF, Williams N, Levin J, Evatt BL, eds. Megakaryocyte development and function. New York: AR Liss, 1986:227-40.

Requests for reprints to: Dr N Pujol-Moix, Servei d'Hematologia, Hospital de la Santa Creu i Sant Pau, Av Pare Claret 167, 08025 Barcelona, Spain. 